\title{
PReS-FINAL-2181: Recombinant il-1ra restores the il-18-nk cell axis in steroid naive systemic juvenile idiopathic arthritis
}

\author{
W De Jager ${ }^{*}$, SJ Vastert, G Mijnheer, BJ Prakken, NM Wulffraat \\ From 20th Pediatric Rheumatology European Society (PReS) Congress \\ Ljubljana, Slovenia. 25-29 September 2013
}

\section{Introduction}

Systemic onset juvenile idiopathic arthritis (sJIA) is an acquired auto-inflammatory disease characterized by systemic inflammation and innate immune activation reflected in uncontrolled production of cytokines such as IL-1, IL-6 and IL-18. In sJIA, NK cell function is severely hampered despite high levels of IL-18. We recently found that defective phosphorylation of the IL-18 receptor beta is responsible for the deficient IL-18-NK cell axis in sJIA.

\section{Objectives}

Given the strong homology between the IL-1 and the IL-18 receptor we questioned whether treatment with rIL-1RA (Anakinra) may directly down regulate IL-18 production and restore the IL-18 signaling cascade in NK cells and, if so, whether this relates to the clinical improvement observed in sJIA patients. Therefore we treated 15 consecutive patients with newly onset sJIA, before start of steroids.

\section{Methods}

Clinical (ACRped) and laboratory parameters (NK Cell activity, cytokine levels) were analyzed during 90 days after initial start of rIL-1RA treatment. To study binding interaction between rIL-1RA and both the IL-1R and the IL-18R a human cell line (KG1) was used.

\section{Results}

We show that patients with sJIA have increased inflammasome activation leading to elevated IL-18 levels. Treatment with recombinant rIL-1RA in steroid naïve newly onset sJIA patients led to rapid resolution of clinical features in $87 \%$ of patients. In vitro, rIL-1RA directly antagonized IL-18 signaling and lead to normalization of both inflammasome activation and IL-18 levels. Finally, in vivo first line treatment with rIL-1RA in sJIA patients led to a normalization of both IL-18 levels and inflammasome activation and a restoration of the deficient IL-18-NK cell axis, correlating with a favorable clinical response in these patients.

\section{Conclusion}

The rapid clinical efficacy of early treatment with rIL-1RA in SIIA is accompanied by a restoration of the IL-18-NKcell axis in sJIA. These data provide biological support for the use of rIL-1RA sJIA patients early in the disease course as first line treatment, as it directly interferes with the deficient IL-18-NK cell axis which may lead to a intrinsically change in the course of the disease.

\section{Disclosure of interest}

None declared.

Published: 5 December 2013

doi:10.1186/1546-0096-11-S2-O16

Cite this article as: De Jager et al:: PReS-FINAL-2181: Recombinant il-1ra restores the il-18-nk cell axis in steroid naive systemic juvenile idiopathic arthritis. Pediatric Rheumatology 2013 11(Suppl 2):O16. 